\title{
Estabilidade de agregados e distribuição de carbono e nutrientes em Argissolo sob adubação orgânica e mineral
}

\author{
Eduardo da Silva Matos( ${ }^{(1)}$, Eduardo de Sá Mendonça(2), Luiz Fernando Carvalho Leite ${ }^{(3)}$ \\ e João Carlos Cardoso Galvão(4)
}

\begin{abstract}
(1)Lehrstuhl für Bodenschutz und Rekultivierung, Brandenburgische Technische Universität Cottbus, Postfach 101344, PLZ 03013 Cottbus, Alemanha. E-mail: matos@tu-cottbus.de (2)Universidade Federal de Viçosa (UFV), Departamento de Solos, Avenida Peter Henry Rolf, s/no,Campus Universitário CEP 36571-000 Viçosa, MG. E-mail: esm@ufv.br ${ }^{(3)}$ Embrapa Meio-Norte, Caixa Postal 001, CEP 64006-220 Teresina, PI. E-mail: luizf@cpamn.embrapa.br (4)UFV, Departamento de Fitotecnia, CEP 36571-000, Viçosa, MG. E-mail: jgalvao@ufv.br
\end{abstract}

Resumo - O objetivo deste trabalho foi avaliar o impacto da adubação orgânica e mineral sobre a estabilidade de agregados e a distribuição de $\mathrm{C}, \mathrm{N}$ e $\mathrm{P}$, em classes de agregados de um Argissolo Vermelho-Amarelo. Os tratamentos consistiram de 0 e $40 \mathrm{~m}^{3} \mathrm{ha}^{-1}$ por ano de adubação orgânica e de 0,250 e $500 \mathrm{~kg} \mathrm{ha}^{-1}$ de adubação mineral N-P-K da fórmula 4-14-8. Uma área sob floresta atlântica foi utilizada como referência. Amostras foram coletadas nas camadas de 0-10 e 10-20 cm. Houve predomínio da classe de agregados entre 4 e $2 \mathrm{~mm}$, que correspondeu a $39,7 \%$ do total de agregados separados por via seca no tratamento com composto orgânico. Os teores de $C$ orgânico total para adubação orgânica foram 17,5 e 36,7\% maiores para as classes 4-2 e 0,105-0,25 mm. A adubação orgânica contribuiu para teores de $\mathrm{N}$ e P totais de 43 e 38,7\% $(0-10 \mathrm{~cm})$ e 35,4 e 36,8\% (10-20 cm), maiores que os dos tratamentos sem adubo orgânico. A relação carbono/nitrogênio se manteve constante entre as classes de agregados de um mesmo tratamento, enquanto a de carbono/fósforo reduziu com o uso de adubo orgânico ou mineral, em relação à mata nativa. Os índices de estabilidade de agregados se correlacionaram positivamente aos teores de carbono orgânico total da classe 4-2 mm.

Termos para indexação: agregação, estrutura do solo, matéria orgânica, sistema de manejo.

\section{Aggregate stability and carbon and nutrients distribution in an Acrisol under organic and mineral fertilization}

\begin{abstract}
The aim of this work was to evaluate the effect of mineral and organic fertilization on the soilwater aggregate stability and on the distribution of $\mathrm{C}, \mathrm{N}$ and $\mathrm{P}$ in different classes of aggregates in an Acrisol. The treatments were 0 and $40 \mathrm{~m}^{3} \mathrm{ha}^{-1}$ per year of organic fertilizer and 0,250 and $500 \mathrm{~kg} \mathrm{ha}^{-1}$ of mineral fertilizer N-P-K of the formula 4-14-8. An area under Atlantic forest was used as reference. Soil samples were collected at $0-10$ and $10-20 \mathrm{~cm}$ depths. The distribution of aggregates showed the highest values from 4 to $2 \mathrm{~mm}$. In the organic fertilizer treatment, the aggregates $4-2 \mathrm{~mm}$ corresponded to $39.7 \%$ of the total on the soil. For the aggregates 4-2 and $0.105-0.25 \mathrm{~mm}$, the total organic carbon contents were 17.5 and $36.7 \%$ higher in the organic fertilizer treatments. The organic fertilization contributed to values of $\mathrm{N}$ and $\mathrm{P}$ of 43 and $38.7 \%$ $(0-10 \mathrm{~cm})$ and 35.4 and $36.8 \%(10-20 \mathrm{~cm})$, which were higher than the treatments without organic fertilizer. The carbon/nitrogen ratio did not vary among the aggregate classes in the same treatment. However, the carbon/ phosphorus ratio was reduced with the use of organic and mineral fertilizer. The indexes of aggregates stability were positively correlated with the content of total organic carbon in the 4-2 $\mathrm{mm}$.
\end{abstract}

Index terms: aggregation, soil structure, organic matter, management system.

\section{Introdução}

A busca por sistemas conservacionistas, que minimizem as emissões de gases do efeito estufa e que contribuam para a manutenção das qualidades física e química do solo, têm relacionado a matéria orgânica (MO) como importante índice na avaliação dos impactos das práticas agrícolas sobre o meio ambiente (Leite et al., 2003). O manejo, o uso e o tempo de utilização promovem diversas alterações nas propriedades do solo, como por exemplo, na estrutura, que está relacionada à agregação. A matéria orgânica é um dos principais agentes de formação e estabilização de agregados, e a diminuição de seu conteúdo no solo pelo cultivo é uma das maiores causas de deterioração da estrutura do solo (Haynes \& Swift, 1990; Wendling

Pesq. agropec. bras., Brasília, v.43, n.9, p.1221-1230, set. 2008 
et al., 2005), já que a partir da agregação, indiretamente, são afetadas as demais características físicas como densidade, porosidade, aeração, capacidade de retenção e infiltração de água (Bayer \& Mielniczuk, 1999).

Correlações positivas entre os teores de carbono (C) orgânico e estabilidade dos agregados em água, de vários tipos de solo, têm sido observadas em diferentes usos e manejo do solo (Wendling et al., 2005; Neves et al., 2006; Ferreira et al., 2007). No entanto, o grau de correlação entre o $\mathrm{C}$ orgânico e agregação depende do clima, tipo de solo, textura e mineralogia. Solos com elevado teor de argila, normalmente, necessitam de maiores teores de $\mathrm{C}$ orgânico para manterem certo nível de agregação e estabilidade de agregados (Douglas \& Goss, 1982). Diferenças no teor de argila causam alterações no regime hídrico do solo e afetam a estabilidade de agregados, que aumenta com a redução do conteúdo de água (Perfect et al., 1990).

Agregados estáveis em água contribuem para melhoria da porosidade, e conseqüentemente, maior infiltração e resistência à erosão. Os agregados não estáveis, quando na superfície, tendem a desaparecer e dispersar-se sob o impacto das gotas de chuva (Assis \& Bahia, 1998). Assim, a utilização de sistemas de cultivo que promovam incremento de $\mathrm{MO}$ do solo podem contribuir para o aumento da estabilidade de agregados e, conseqüentemente, para a melhoria da qualidade física do solo.

Este trabalho teve como objetivo quantificar o impacto da adubação orgânica e química sobre a estabilidade dos agregados em água e sobre a distribuição de $\mathrm{C}, \mathrm{N}$ e $\mathrm{P}$, nas diferentes classes de agregados de um Argissolo Vermelho-Amarelo.

\section{Material e Métodos}

O estudo foi realizado em experimento de campo de longa duração, com diferentes sistemas de manejo, na Estação Experimental da Universidade Federal de Viçosa, no Município de Coimbra, MG (2045'S, $42^{\circ} 51^{\prime} \mathrm{W}$ e $700 \mathrm{~m}$ de altitude). A temperatura média anual é de $19^{\circ} \mathrm{C}$, e a precipitação média anual de $1.350 \mathrm{~mm}$, concentrada nos meses de outubro a março (Galvão, 1995).

O solo do experimento é um Argissolo VermelhoAmarelo distrófico, fase terraço, muito argiloso, que apresentou, no início do experimento, na camada de 0-20 cm: $\mathrm{pH}\left(\mathrm{H}_{2} \mathrm{O}\right)$ 5,9; $\mathrm{Al}^{3+}, \mathrm{Ca}^{2+}$ e $\mathrm{Mg}^{2+}$ de 0, 2,6 e $1,7 \mathrm{cmol}_{\mathrm{c}} \mathrm{dm}^{-3}$, respectivamente; $\mathrm{P}$ e $\mathrm{K}$ disponíveis
(Mehlich-1) de 11 e $58 \mathrm{mg} \mathrm{dm}^{-3}$, respectivamente; e teor de C orgânico de 2,3 dag $\mathrm{kg}^{-1}$ (Walkey-Black). Os teores de areia grossa, areia fina, silte e argila foram 80, 60, 160 e $700 \mathrm{~g} \mathrm{~kg}^{-1}$, respectivamente.

Originalmente sob vegetação natural até 1930, a área passou a ser cultivada subseqüentemente, por 53 anos, com culturas de subsistência como o milho e o feijão. O experimento foi iniciado em 1984/1985, com cultivo de milho exclusivamente, e os tratamentos foram formados a partir de combinações de dois níveis de composto orgânico, nas doses $0 \mathrm{e} 40 \mathrm{~m}^{3} \mathrm{ha}^{-1}$ por ano e três doses de adubo mineral N-P-K, nas doses 0,250 e $500 \mathrm{~kg} \mathrm{ha}^{-1}$ por ano da fórmula 4-14-8, aplicados no plantio. Foram realizadas adubações de cobertura com sulfato de amônio, nas doses 0,100 e $200 \mathrm{~kg} \mathrm{ha}^{-1}$, exceto para os tratamentos que continham apenas adubo orgânico. Produzido com palha de soja e de feijão misturados a esterco bovino, o adubo orgânico utilizado apresentou, em média, $380 \mathrm{~g} \mathrm{~kg}^{-1}$ de água, $0,36 \mathrm{~g} \mathrm{~cm}^{-3} \mathrm{de}$ densidade, teores de $\mathrm{P}, \mathrm{K}, \mathrm{Ca}, \mathrm{Mg}$ e $\mathrm{N}$ de $0,7,2,8,1,0,0,4$ e 3,2 dag kg-1 , respectivamente, e relação $\mathrm{C} / \mathrm{N}$ igual a 5 . $\mathrm{O}$ composto orgânico e o adubo químico foram aplicados ao sulco de semeadura e incorporados com enxada.

O delineamento experimental utilizado foi o de blocos ao acaso, em arranjo fatorial 2x3 (duas doses de adubação orgânica e três doses de adubação mineral), com quatro repetições, no total de 24 unidades experimentais.

Uma área de floresta atlântica, próxima ao experimento (150 m), sob o mesmo tipo de solo, foi utilizada como padrão de referência. Nessa área de mata, foi definido um transecto de aproximadamente $100 \mathrm{~m}$, no qual se delimitaram quatro repetições.

Amostras de solo foram coletadas das camadas de 0-10 e 10-20 cm. A distribuição dos agregados por via seca foi avaliada por meio da separação das classes de agregados, em conjunto de peneiras com malhas nos diâmetros 2, 1, 0,5, 0,25, e 0,105 $\mathrm{mm}$, tendo-se utilizado aparelho Produtest (intensidade 5) submetido a 5 min de vibração.

Para a avaliação da estabilidade de agregados em água, amostras de $25 \mathrm{~g}$ de grânulos entre 4 e $2 \mathrm{~mm}$ foram previamente submetidas a umedecimento lento e, em seguida, colocadas em conjunto de peneiras com malhas de diâmetros $2,1,0,5,0,25$, e $0,105 \mathrm{~mm}$, imersas em água e agitadas por um oscilador mecânico de Yoder, de freqüência igual a 32 ciclos $\min ^{-1}$ e $4 \mathrm{~cm}$ de amplitude vertical, durante $15 \mathrm{~min}$ (Kemper \& Chepil, 1965). 
Após peneiramento em água, obteve-se a proporção de cada classe de agregado, em relação ao total, e se procedeu ao cálculo do diâmetro médio ponderado (DMP) e do diâmetro médio geométrico (DMG), a partir das fórmulas:

$\mathrm{DMP}=\sum_{\mathrm{i}=1}^{\mathrm{n}}(\mathrm{wi} \cdot \mathrm{xi})$

$\mathrm{DMG}=10^{\sum_{\mathrm{i}=1}^{\mathrm{n}}(\mathrm{wi} \log \mathrm{xi}) / \sum_{\mathrm{i}=1}^{\mathrm{n}} \mathrm{wi}}$

em que: wi é a proporção de agregados nas diferentes classes de peneiras, e xi é o valor médio de cada classe (mm).

Para avaliação dos teores $\mathrm{de} \mathrm{C}, \mathrm{Ne} \mathrm{P}$, foram utilizadas amostras dos agregados separados por via seca nas classes $4-2,2-1,1-0,5,0,5-0,25$ e $0,25-0,105 \mathrm{~mm}$. O carbono orgânico total (COT) foi determinado por meio da oxidação úmida com dicromato de potássio, em meio ácido forte, com fonte externa de calor (Yeomans \& Bremner, 1988). O fósforo total foi extraído por digestão nitroperclórica $\left(\mathrm{HNO}_{3}\right.$ e $\mathrm{HClO}_{4}$ concentrado), de acordo com Embrapa (1997), e determinado por meio de reação com ácido ascórbico, em espectrofotômetro de absorção molecular, com comprimento de onda de $882 \mathrm{~nm}$ (Murphy \& Riley, 1962). Para determinação de $\mathrm{N}$ total, as amostras foram submetidas à digestão sulfúrica e $\mathrm{N}$ dosado por meio de destilação Kjeldahl (Bremner \& Mulvaney, 1982).

Os dados obtidos foram submetidos à análise de variância, tendo-se empregado os testes F e de Tukey, a $5 \%$ de probabilidade.

\section{Resultados e Discussão}

$\mathrm{Na}$ distribuição dos agregados por via seca, houve predominância da classe de agregados 4-2 mm nas camadas de 0-10 e 10-20 cm (Figura 1). Para o tratamento que recebeu o composto orgânico, essa fração correspondeu a 39,6\% dos agregados do solo na camada de $0-10 \mathrm{~cm}$. A classe de agregados menores que $0,105 \mathrm{~mm}$ apresentou valores médios de 3,7\% nos tratamentos estudados (Figura 1) e de 3,1\% na mata nativa. $\mathrm{O}$ uso do adubo mineral, na dose $250 \mathrm{~kg} \mathrm{ha}^{-1}$, não alterou a distribuição de agregados no solo, em comparação com o tratamento sem adubação. Entretanto, a adubação mineral, na dose $500 \mathrm{~kg} \mathrm{ha}^{-1}$, apresentou valores cerca de $6 \%$ menores de macroagregados entre 4-2 $\mathrm{mm}$, em relação à adubação orgânica na camada de $0-10 \mathrm{~cm}$.
Foram observados maiores teores de COT, nas classes de agregados separados por via seca para os tratamentos que receberam composto orgânico (Tabela 1). Em média, na classe de 4-2 mm e camada de 0-10 cm, os valores de COT foram 17,5\% maiores nos tratamentos com adição de composto orgânico, do que aqueles que não receberam o adubo orgânico. Em geral, houve aumento deste percentual nos agregados menores. A classe de 0,105-0,250 $\mathrm{mm}$ apresentou a maior diferença percentual, com valores de COT 36,7\% maiores que os dos tratamentos com adubação mineral (Tabela 1). Esses dados corroboram os de Passos et al. (2007), que observaram aumento dos teores de COT com a redução do tamanho dos agregados no solo e os relacionaram à maior associação da fração argila com a MO do solo, em razão dos maiores teores de argila em microagregados do que em macroagregados.

Entretanto, alguns autores têm observado teores mais elevados de COT nas classes de agregados maiores que $2 \mathrm{~mm}$ do que para classes menores (Perin et al., 2002). Six et al. (2000) verificaram valores de $\mathrm{C}$ em macroagregados cerca de 1,65 vez maiores que nos microagregados, em que a mineralogia de argilas 2:1 prevalecia, e não constataram diferenças no teor de $\mathrm{C}$ para micro e macroagregados em solos em que a mineralogia de argilas 1:1 prevalecia. Em solos com mineralogia de argila 2:1 em maior proporção, a MO pode ser considerada o principal agente agregante no solo, que atua pela formação de pontes entre os complexos orgânicos polivalentes e as cargas negativas das argilas. Contudo, em solos com mineralogia dominada por argilas 1:1 e óxidos, a MO passa a não ser o principal agente agregante, pois podem ocorrer atrações eletrostáticas entre óxidos e as lâminas de silicatos, em razão da presença simultânea de cargas positivas e negativas (Six et al., 2000).

Em média, o nitrogênio total (NT) foi 43 e 35,4\% maior nos tratamentos que receberam adubação orgânica, em todas as classes de agregados nas camadas de $0-10$ e 10-20 cm, respectivamente (Tabela 2). Os maiores valores de NT estão relacionados aos maiores conteúdos de MO, pois aproximadamente $95 \%$ do N no solo está relacionado às formas orgânicas (Stevenson, 1994).

Seguindo a mesma tendência do COT, o P total $(\mathrm{Pt})$ apresentou teores mais elevados nos tratamentos que receberam adubação orgânica (Tabela 3) e esteve relacionado aos maiores aportes de $\mathrm{P}$, em conseqüência do uso do adubo orgânico (100,8 $\mathrm{kg} \mathrm{ha}^{-1}$ por ano). 
A adubação mineral também contribuiu para os maiores teores totais de $\mathrm{P}$, nas diferentes classes de agregados no solo, em comparação ao tratamento que não recebeu adubação química. A interação adubação orgânica com adubação mineral não apresentou efeito significativo quanto às concentrações de Pt. Entre as classes de agregados, os maiores valores de Pt foram observados na classe de agregados $0,25-0,105 \mathrm{~mm}$, nas camadas de 0-10 cm (941,6 $\left.\mathrm{mg} \mathrm{kg}^{-1}\right)$ e 10-20 cm (907 $\left.\mathrm{mg} \mathrm{kg}^{-1}\right)$. Entretanto, a classe de 4-2 $\mathrm{mm}$ apresentou os menores valores, 830 e $772,2 \mathrm{mg} \mathrm{kg}^{-1}$ para $0-10$ e $10-20 \mathrm{~cm}$, respectivamente. Adesodun et al. (2007) também observaram incremento das concentrações de $\mathrm{N}$ e $\mathrm{P}$ em microagregados de solos cultivados. O aumento da concentração de $\mathrm{P}$ em microagregados pode estar relacionado ao conteúdo de MO mais elevado, possivelmente com maiores teores de frações orgânicas de P.

De forma geral, a relação $\mathrm{C} / \mathrm{N}$ manteve-se constante, entre as diferentes classes de agregados de um mesmo tratamento (Figura 2), similarmente ao observado por Passos et al., (2007). A adubação orgânica contribuiu para os menores valores da relação $\mathrm{C} / \mathrm{N}$, em comparação com a adubação mineral, apenas na profundidade $0-10 \mathrm{~cm}$. Isto pode acarretar aumento da dinâmica de $\mathrm{N}$ e $\mathrm{C}$ no solo. A melhoria da qualidade da matéria orgânica
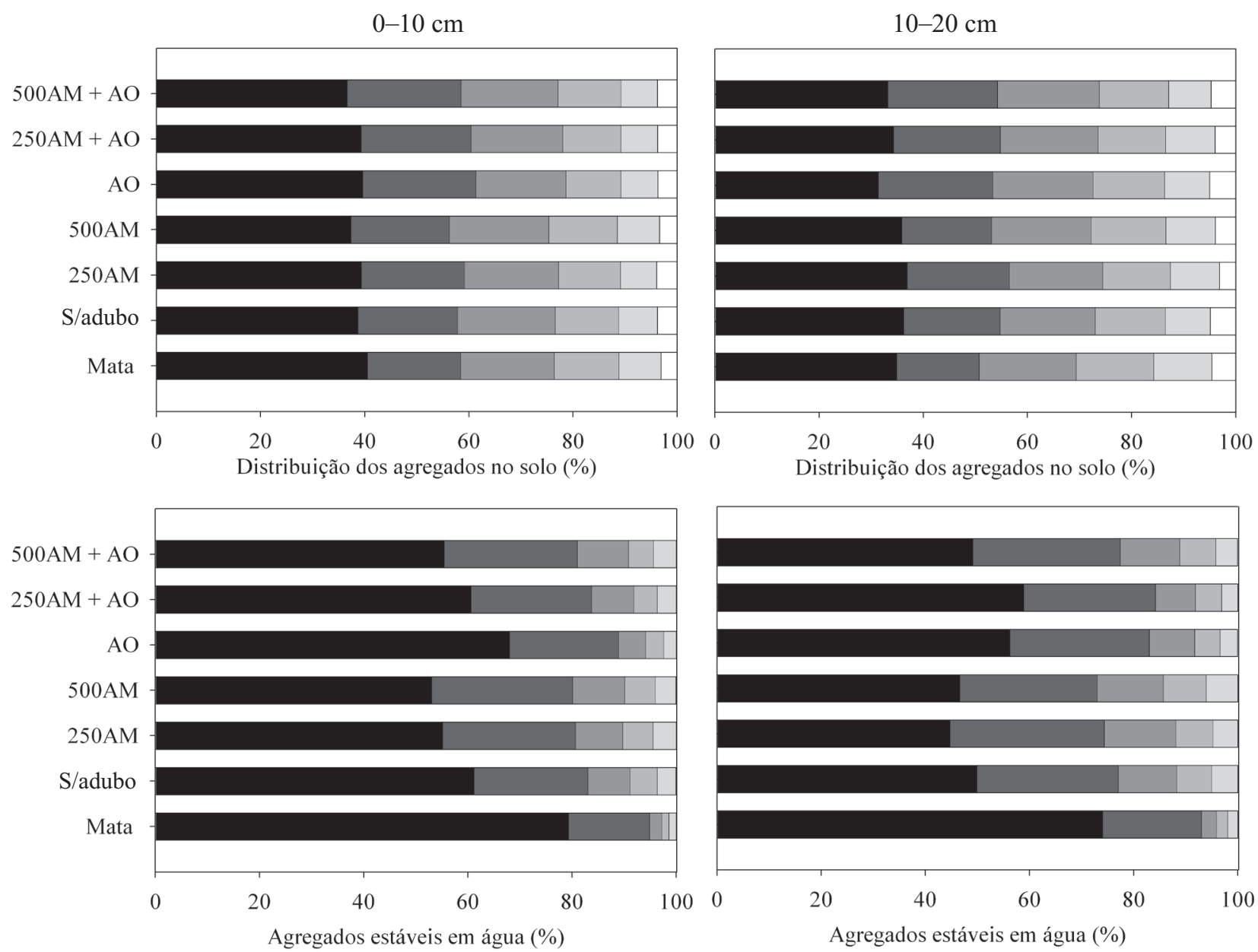

\begin{tabular}{|lll|}
\hline$\square-2 \mathrm{~mm}$ & $\square 0,50 \mathrm{~mm}$ & $\square 0,25-0,105 \mathrm{~mm}$ \\
$2-1 \mathrm{~mm}$ & $\square 0,50-0,25 \mathrm{~mm}$ & $\square<0,105 \mathrm{~mm}$ \\
\hline
\end{tabular}

Figura 1. Percentagem da distribuição de agregados no solo e de agregados estáveis em água, nas camadas de 0-10 e 10-20 cm. AO, adubação orgânica; AM, adubação mineral de 0, 250 e $500 \mathrm{~kg} \mathrm{ha}^{-1}$ da fórmula 4-14-8. Para agregados estáveis em água a menor classe de agregados apresentada é de 0,25-0,105 mm. 
contribui para a melhoria da estabilidade dos agregados, que está mais relacionada a mudanças nos constituintes orgânicos do que ao conteúdo total de MO (Tisdall \& Oades, 1982; Carpenedo \& Mielniczuk, 1990).

Houve tendência de redução da relação $\mathrm{C} / \mathrm{P}$ (Figura 2), principalmente nas classes de agregados maiores que $1 \mathrm{~mm}$, quando se utilizou o composto orgânico (20 e 11,9\% nas camadas 0-10 e 10-20 cm, respectivamente), em relação ao tratamento sem adubo. A mesma tendência de redução da relação $\mathrm{C} / \mathrm{P}$ foi observada nos tratamentos que receberam adubação mineral, porém com menor resposta que a adubação orgânica: 10 e 10,1\% para a adubação com 250 e $500 \mathrm{~kg}$ de 4-14-8, respectivamente, na camada de $0-10 \mathrm{~cm}$. Esse maior aumento de $\mathrm{P}$, em comparação ao conteúdo total de $\mathrm{C}$, proporcionou a redução da relação $\mathrm{C} / \mathrm{P}$ e pode estar relacionado, não só à fração inorgânica de $\mathrm{P}$, como ao aumento da fração de $\mathrm{P}$ orgânico. Nesse mesmo experimento, foi observada a tendência da relação $\mathrm{C} / \mathrm{P}$ orgânico de manter-se constante, entre os tratamentos, tendo-se constatado aumento dos valores de P orgânico com o

Tabela 1. Teores totais de carbono orgânico (COT, $\mathrm{g} \mathrm{kg}^{-1}$ ) em Argissolo sob mata nativa, e com adubação orgânica e adubação mineral, nas camadas de 0-10 e 10-20 cm, nas diferentes classes de $\operatorname{agregados}^{(1)}$.

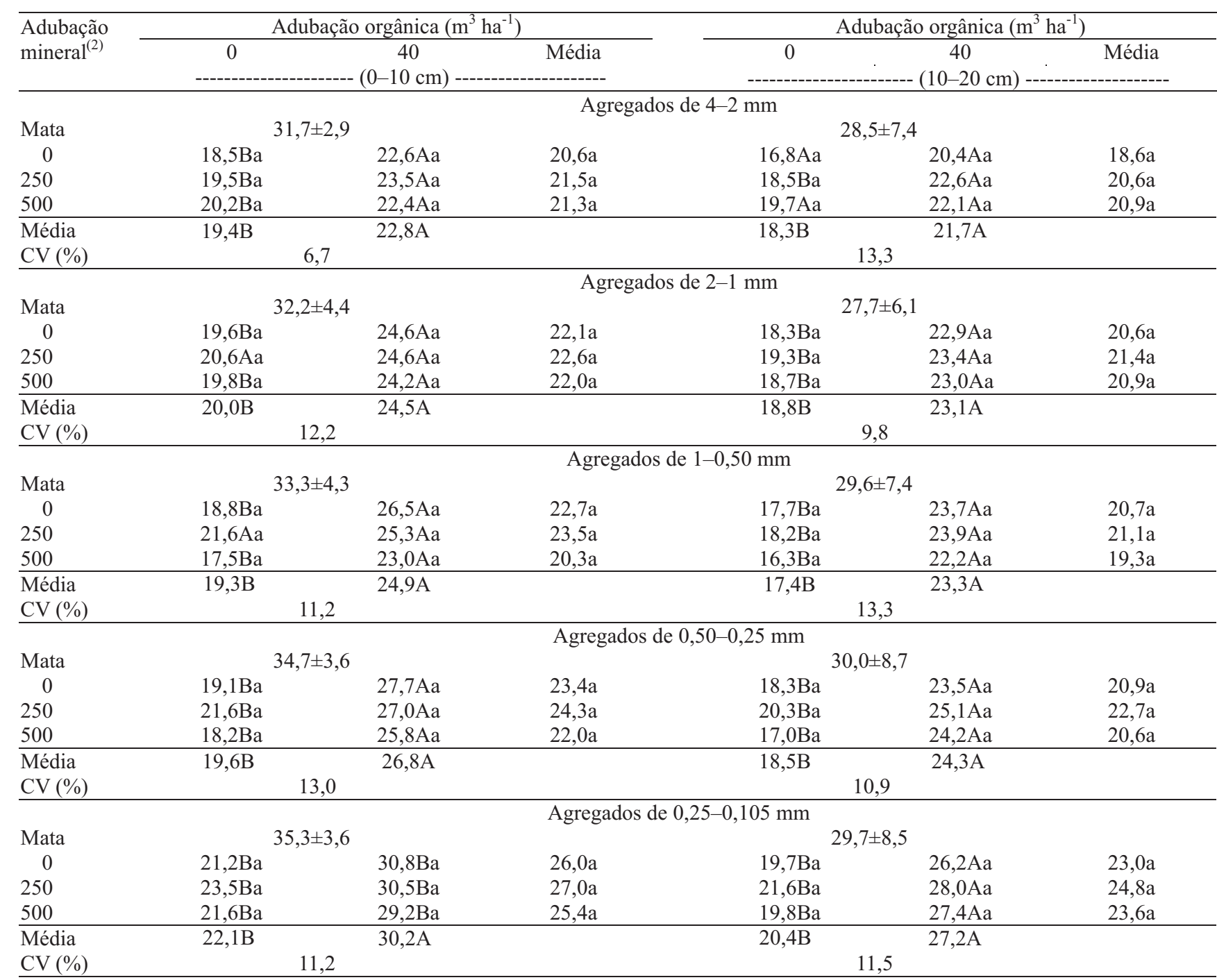

${ }^{(1)}$ Médias seguidas por letras iguais, maiúsculas na linha e minúsculas na coluna, não diferem entre si pelo teste de Tukey, a $5 \%$ de probabilidade. ${ }^{(2)}$ Adubação mineral de 0,250 e $500 \mathrm{~kg} \mathrm{ha}^{-1}$ da fórmula 4-14-8. 
aumento do teor de COT no solo (Matos et al., 2006). Segundo Zhang \& Mackenzie (1997), o reservatório de $\mathrm{P}$ orgânico tende a aumentar, quando o manejo do solo favorece o acúmulo de $\mathrm{C}$ com adições freqüentes de fertilizantes, e diminui em solos intensamente cultivados ou manejados inadequadamente, onde se tem baixa reposição do $\mathrm{P}$ exportado.

A maior proporção de agregados estáveis em água ocorreu na classe 4-2 mm, em ambas as profundidades estudadas (Figura 1). O solo sob mata apresentou maiores valores desta classe, que corresponderam a 79,4 e $74,1 \%$ dos agregados estáveis em água, nas camadas de $0-10$ e $10-20 \mathrm{~cm}$, respectivamente. Os tratamentos que receberam apenas adubação mineral apresentaram os menores valores de agregados estáveis, em ambas as profundidades. Esses resultados estão de acordo com Neves et al. (2006), que observaram redução da estabilidade de agregados estáveis, em solos submetidos à adubação mineral, em comparação aos solos sob floresta.

Entre os tratamentos de adubação, o composto orgânico propiciou maior proporção da classe

Tabela 2. Teores totais de nitrogênio (NT, $\mathrm{g} \mathrm{kg}^{-1}$ ) em Argissolo sob mata nativa, e com adubação orgânica e adubação mineral, nas camadas de $0-10$ e $10-20 \mathrm{~cm}$, nas diferentes classes de $\operatorname{agregados}^{(1)}$.

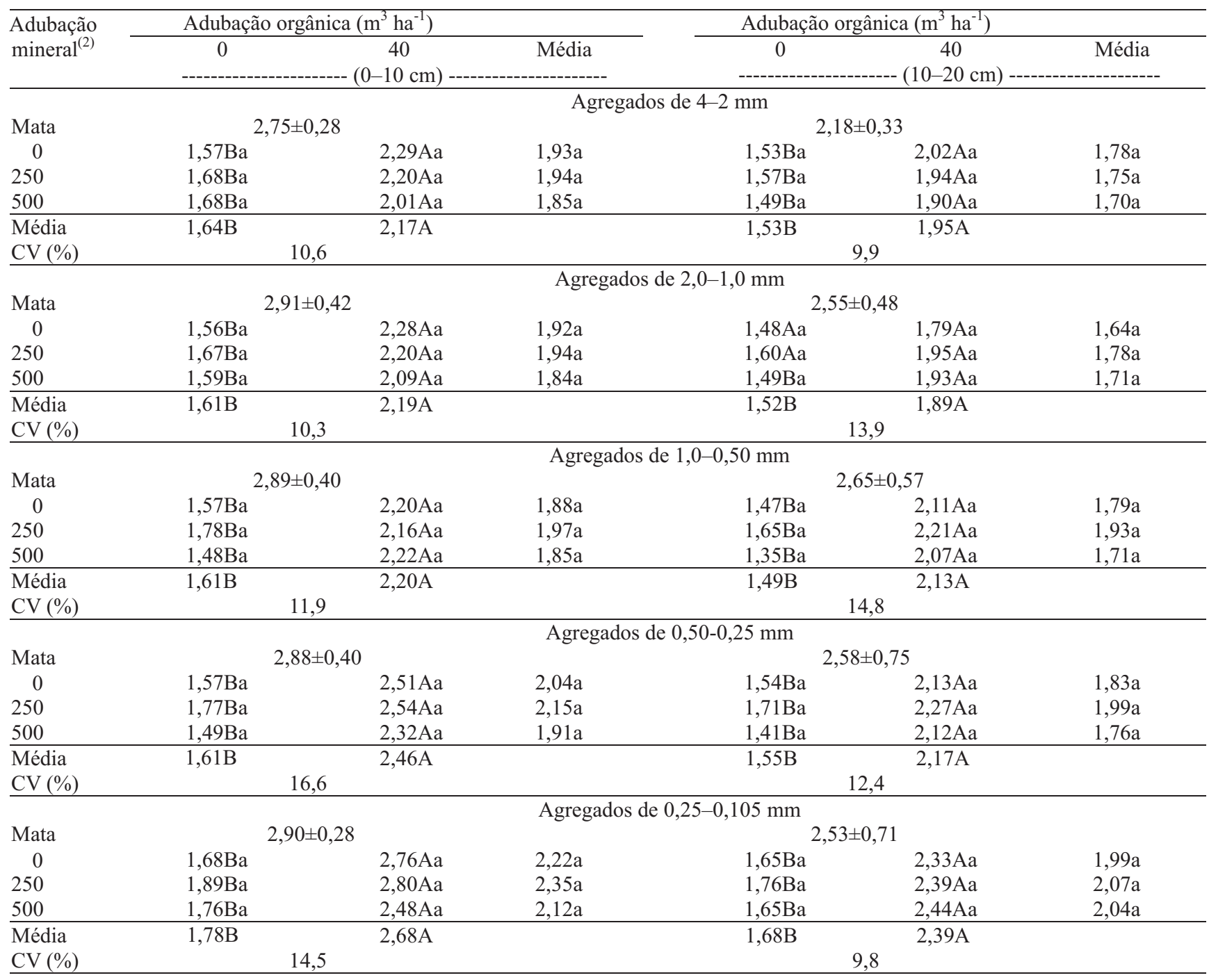

${ }^{(1)}$ Médias seguidas por letras iguais, maiúsculas na linha e minúsculas na coluna, não diferem entre si pelo teste de Tukey, a $5 \%$ de probabilidade. ${ }^{(2)}$ Adubação mineral de 0,250 e $500 \mathrm{~kg} \mathrm{ha}^{-1}$ da fórmula 4-14-8. 
4-2 mm estável em água, correspondente a $68 \%$ dos agregados na profundidade de $0-10 \mathrm{~cm}$, o que pode estar relacionado ao maior aporte de $\mathrm{MO}$ promovido por este sistema de manejo. Esses resultados corroboram a afirmativa de Haynes \& Swift (1990) de que a incorporação de resíduos orgânicos, nas camadas aráveis, promove aumento do conteúdo de MO no solo e proporciona aumento da estabilidade de agregados.

Os índices de agregação DMP e o DMG variaram de 2,08 a 2,64 e de 1,72 a 2,44 na camada de $0-10 \mathrm{~cm}$, e os de 1,93 a 2,54 e de 1,53 a 2,29 na camada de 10-20 cm, respectivamente (Tabela 4). A mata apresentou valores mais elevados de DMP e DMG, em ambas as profundidades. Wendling et al. (2005) também observaram índices de agregação mais elevados em mata nativa, em comparação com diversos sistemas de cultivo do solo, que estiveram relacionados aos maiores conteúdos de COT e à ausência de histórico de revolvimento do solo.

Entre os sistemas de adubação empregados, observaram-se maiores valores de DMP e DMG apenas

Tabela 3. Teores totais de fósforo $\left(\mathrm{Pt}, \mathrm{mg} \mathrm{kg}^{-1}\right)$ em Argissolo sob mata nativa, e com adubação orgânica e adubação mineral, nas camadas de $0-10$ e 10-20 cm, nas diferentes classes de $\operatorname{agregados}^{(1)}$.

\begin{tabular}{|c|c|c|c|c|c|c|}
\hline \multirow{3}{*}{$\begin{array}{l}\text { Adubação } \\
\text { mineral }^{(2)}\end{array}$} & \multicolumn{3}{|c|}{ Adubação orgânica $\left(\mathrm{m}^{3} \mathrm{ha}^{-1}\right)$} & \multicolumn{3}{|c|}{ Adubação orgânica $\left(\mathrm{m}^{3} \mathrm{ha}^{-1}\right)$} \\
\hline & 0 & 40 & Média & \multirow{2}{*}{0} & 40 & \multirow{2}{*}{ Média } \\
\hline & --------------. & $(0-10 \mathrm{~cm})-$ & ---------- & & $--(10-20 \mathrm{cl}$ & \\
\hline & \multicolumn{6}{|c|}{ Agregados de 4-2 mm } \\
\hline Mata & \multicolumn{2}{|c|}{$562,4 \pm 25,3$} & & \multicolumn{2}{|c|}{$526,1 \pm 19,9$} & \\
\hline 0 & $614,9 \mathrm{Ba}$ & $894,9 \mathrm{Aa}$ & $754,9 b$ & $621,0 \mathrm{Ba}$ & $833,2 \mathrm{Aa}$ & $727,1 \mathrm{a}$ \\
\hline 250 & $747,0 \mathrm{Ba}$ & $995,7 \mathrm{Aa}$ & $871,3 \mathrm{a}$ & $719,6 \mathrm{Ba}$ & $879,3 \mathrm{Aa}$ & $799,4 a$ \\
\hline 500 & $744,9 \mathrm{Ba}$ & $982,6 \mathrm{Aa}$ & $863,7 \mathrm{a}$ & $649,8 \mathrm{Ba}$ & $930,2 \mathrm{Aa}$ & $790,0 \mathrm{a}$ \\
\hline Média & $702,2 \mathrm{~B}$ & $957,7 \mathrm{~A}$ & & $663,5 \mathrm{~B}$ & $880,9 \mathrm{~A}$ & \\
\hline \multirow[t]{2}{*}{ CV $(\%)$} & \multicolumn{2}{|c|}{9,6} & \multicolumn{4}{|c|}{8,5} \\
\hline & \multirow{2}{*}{\multicolumn{2}{|c|}{$665,1 \pm 30,3$}} & \multicolumn{3}{|c|}{ Agregados de $2-1 \mathrm{~mm}$} & \\
\hline Mata & & & & & & \\
\hline 0 & $673,8 \mathrm{Ba}$ & $984,2 \mathrm{Aa}$ & $829,0 \mathrm{a}$ & $657,0 \mathrm{Ba}$ & $886,0 \mathrm{Aa}$ & $771,4 b$ \\
\hline 250 & $766,5 \mathrm{Ba}$ & $1.070,6 \mathrm{Aa}$ & $918,5 \mathrm{a}$ & $743,9 \mathrm{Ba}$ & $1.034,6 \mathrm{Aa}$ & $889,2 \mathrm{a}$ \\
\hline 500 & $749,9 \mathrm{Ba}$ & $1.088,9 \mathrm{Aa}$ & $919,3 \mathrm{a}$ & $697,9 \mathrm{Ba}$ & $1.024,9 \mathrm{Aa}$ & $861,4 \mathrm{ab}$ \\
\hline Média & $730,0 \mathrm{~B}$ & $1.047,9 \mathrm{~A}$ & & $699,6 \mathrm{~B}$ & $981,8 \mathrm{~A}$ & \\
\hline \multirow[t]{2}{*}{ CV $(\%)$} & \multicolumn{2}{|c|}{9,3} & \multicolumn{4}{|c|}{9,9} \\
\hline & \multicolumn{6}{|c|}{ Agregados de $1-0,50 \mathrm{~mm}$} \\
\hline Mata & \multicolumn{2}{|c|}{$612,3 \pm 14,4$} & \multicolumn{4}{|c|}{$562,9 \pm 20,7$} \\
\hline 0 & $673,7 \mathrm{Ba}$ & $963,6 \mathrm{Aa}$ & $818,6 \mathrm{a}$ & $705,5 \mathrm{Ba}$ & $915,8 \mathrm{Aa}$ & $810,6 a$ \\
\hline 250 & $822,3 \mathrm{Ba}$ & $1.058,0 \mathrm{Aa}$ & $940,1 \mathrm{a}$ & $786,9 \mathrm{Ba}$ & $1.025,1 \mathrm{Aa}$ & $906,0 \mathrm{a}$ \\
\hline 500 & $738,5 \mathrm{Ba}$ & $1.067,8 \mathrm{Aa}$ & $903,2 \mathrm{a}$ & $689,6 \mathrm{Ba}$ & $1.021,6 \mathrm{Aa}$ & $855,6 \mathrm{a}$ \\
\hline Média & $744,8 \mathrm{~B}$ & $1.029,8 \mathrm{~A}$ & & $727,3 \mathrm{~B}$ & $987,5 \mathrm{~A}$ & \\
\hline \multirow[t]{2}{*}{ CV $(\%)$} & \multicolumn{2}{|c|}{11,9} & \multicolumn{4}{|c|}{11,9} \\
\hline & \multicolumn{6}{|c|}{ Agregados de $0,50-0,25 \mathrm{~mm}$} \\
\hline Mata & \multicolumn{2}{|c|}{$690,1 \pm 68,9$} & \multicolumn{4}{|c|}{$604,6 \pm 23,7$} \\
\hline 0 & $722,9 \mathrm{Ba}$ & $995,9 \mathrm{Aa}$ & $859,4 b$ & $714,0 \mathrm{Ba}$ & $934,8 \mathrm{Aa}$ & $824,4 a$ \\
\hline 250 & $865,3 \mathrm{Ba}$ & $1.107,2 \mathrm{Aa}$ & $986,2 \mathrm{a}$ & $779,1 \mathrm{Ba}$ & $1.090,7 \mathrm{Aa}$ & $934,9 \mathrm{a}$ \\
\hline 500 & $781,3 \mathrm{Ba}$ & $1.020,9 \mathrm{Aa}$ & $901,1 \mathrm{ab}$ & $727,6 \mathrm{Ba}$ & $980,8 \mathrm{Aa}$ & $854,2 \mathrm{a}$ \\
\hline Média & $789,8 \mathrm{~B}$ & $1.041,3 \mathrm{~A}$ & & $740,2 \mathrm{~B}$ & $1.002,1 \mathrm{~A}$ & \\
\hline \multirow[t]{2}{*}{ CV $(\%)$} & \multicolumn{2}{|c|}{9,9} & & & & \\
\hline & & & Agregad & $105 \mathrm{~mm}$ & & \\
\hline Mata & & & & & & \\
\hline 0 & $663,1 \mathrm{Bb}$ & $1.019,2 \mathrm{Aa}$ & $841,2 b$ & $685,0 \mathrm{Ba}$ & $961,1 \mathrm{Aa}$ & $823,2 b$ \\
\hline 250 & $838,7 \mathrm{Ba}$ & $1.153,7 \mathrm{Aa}$ & $996,2 \mathrm{a}$ & $799,6 \mathrm{Ba}$ & $1.096,4 \mathrm{Aa}$ & $948,0 \mathrm{a}$ \\
\hline 500 & $820,4 \mathrm{Bab}$ & $1.154,1 \mathrm{Aa}$ & $987,3 \mathrm{a}$ & $785,3 \mathrm{Ba}$ & $1.114,5 \mathrm{Aa}$ & $949,9 \mathrm{a}$ \\
\hline Média & $774,1 \mathrm{~B}$ & $1.109,0 \mathrm{~A}$ & & $756,6 \mathrm{~B}$ & $1.057,3 \mathrm{~A}$ & \\
\hline CV (\%) & & & & & & \\
\hline
\end{tabular}

${ }^{(1)}$ Médias seguidas por letras iguais, maiúsculas na linha e minúsculas na coluna, não diferem entre si pelo teste de Tukey, a 5\% de probabilidade. ${ }^{(2)}$ Adubação mineral de 0,250 e $500 \mathrm{~kg} \mathrm{ha}^{-1}$ da fórmula 4-14-8. 
na camada de $10-20 \mathrm{~cm}$, nos tratamentos que receberam o composto orgânico. Na camada de $0-10 \mathrm{~cm}$, houve redução do DMP com o aumento da dose de adubo utilizada. Os maiores valores de DMP e DMG são atribuídos à maior quantidade de agregados maiores que $2 \mathrm{~mm}$, e esta maior agregação pode estar relacionada ao maior acúmulo de $\mathrm{MO}$, proporcionado pelos sistemas de manejo em que não foi utilizada a adubação mineral.
Os índices de agregação correlacionaram-se positivamente aos teores de COT $(\mathrm{p}<0.01)$ da classe de agregados utilizada para a avaliação da estabilidade de agregados (4-2 mm). Diversos trabalhos associam os maiores valores de DMP e DMG aos sistemas de manejo que promovem o incremento de $\mathrm{MO}$ e contribuem para o aumento da agregação do solo (Palmeira et al., 1999; Perin et al., 2002; Bastos et al., 2005).
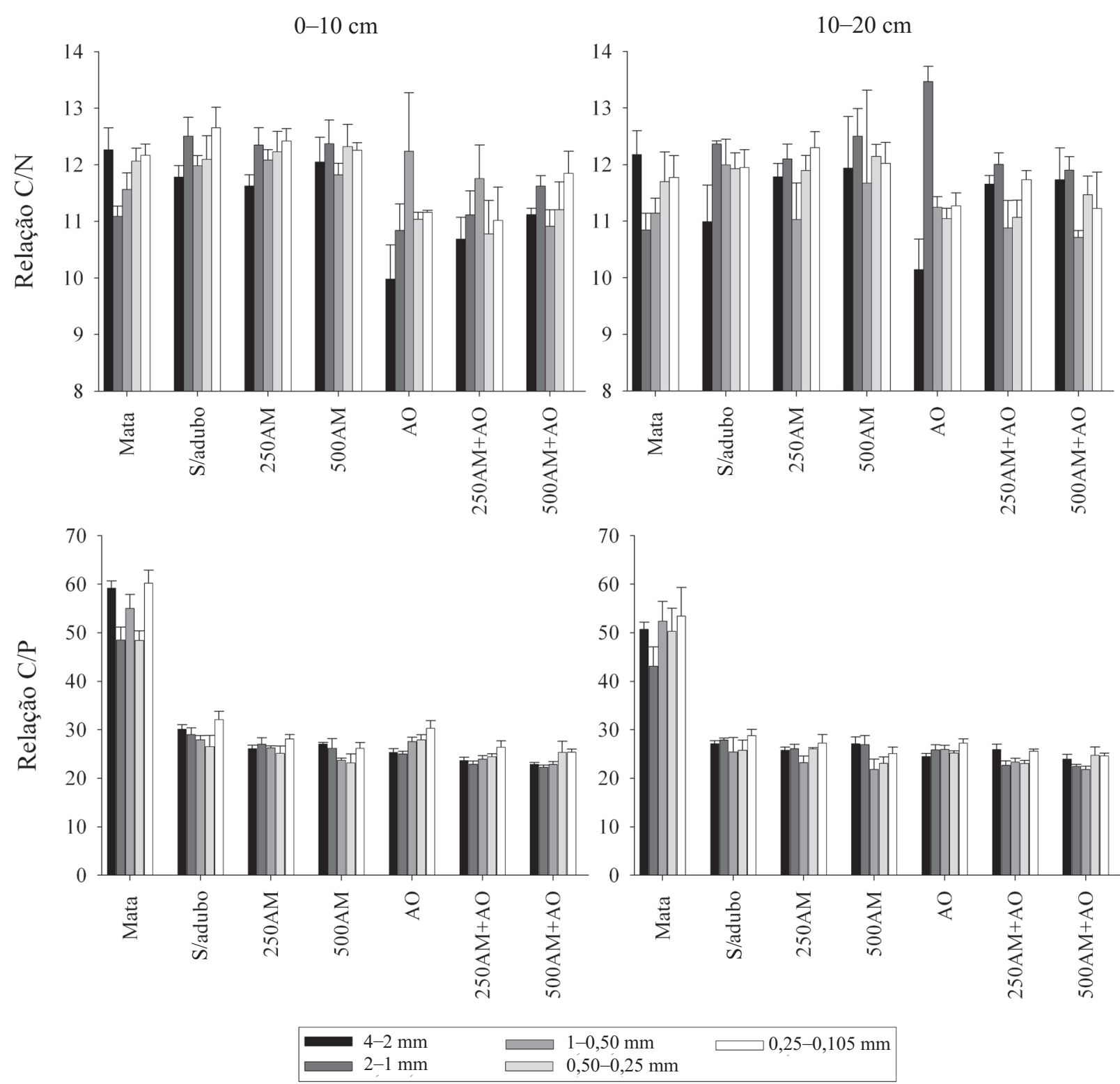

Figura 2. Relação $\mathrm{C} / \mathrm{N}$ e $\mathrm{C} / \mathrm{P}$ dos agregados do solo, nas profundidades de $0-10$ e $10-20 \mathrm{~cm}$. AO, adubação orgânica; $\mathrm{AM}$, adubação mineral de 0,250 e $500 \mathrm{~kg} \mathrm{ha}^{-1}$ da fórmula 4-14-8. 
Tabela 4. Diâmetro médio ponderado (DMP) e diâmetro médio geométrico (DMG), ambos em mm, em Argissolo sob mata nativa, e com adubação orgânica e adubação mineral, nas camadas de $0-10$ e $10-20 \mathrm{~cm}^{(1)}$.

\begin{tabular}{|c|c|c|c|c|c|c|}
\hline \multirow{2}{*}{$\begin{array}{l}\text { Adubação } \\
\text { mineral }^{(2)}\end{array}$} & \multicolumn{3}{|c|}{ Adubação orgânica $\left(\mathrm{m}^{3} \mathrm{ha}^{-1}\right)$} & \multicolumn{3}{|c|}{ Adubação orgânica $\left(\mathrm{m}^{3} \mathrm{ha}^{-1}\right)$} \\
\hline & 0 & 40 & Média & 0 & 40 & Média \\
\hline & \multicolumn{6}{|c|}{ Camada de $0-10 \mathrm{~cm}$} \\
\hline Mata & \multicolumn{3}{|c|}{$2,64 \pm 0,13$} & \multicolumn{2}{|c|}{$2,44 \pm 0,12$} & \\
\hline 0 & $2,25 \mathrm{Aa}$ & 2,41Aa & $2,33 a$ & $1,87 \mathrm{Aa}$ & $2,10 \mathrm{Aa}$ & $1,99 \mathrm{a}$ \\
\hline 250 & $2,08 \mathrm{Aa}$ & $2,25 \mathrm{Aa}$ & $2,17 \mathrm{ab}$ & $1,75 \mathrm{Aa}$ & $1,88 \mathrm{Aa}$ & $1,82 \mathrm{a}$ \\
\hline 500 & $2,10 \mathrm{Aa}$ & $2,15 \mathrm{Aa}$ & $2,12 b$ & $1,72 \mathrm{Aa}$ & $1,77 \mathrm{Aa}$ & $1,75 \mathrm{a}$ \\
\hline Média & $2,15 \mathrm{~A}$ & $2,27 \mathrm{~A}$ & & $1,78 \mathrm{~A}$ & $1,92 \mathrm{~A}$ & \\
\hline $\mathrm{CV}(\%)$ & \multicolumn{2}{|c|}{6,5} & \multicolumn{4}{|c|}{10,6} \\
\hline & \multicolumn{6}{|c|}{ Camada de $10-20 \mathrm{~cm}$} \\
\hline Mata & \multicolumn{2}{|c|}{$2,54 \pm 0,17$} & \multicolumn{4}{|c|}{$2,29 \pm 0,16$} \\
\hline 0 & $2,02 \mathrm{Aa}$ & $2,18 \mathrm{Aa}$ & $2,10 \mathrm{a}$ & $1,62 \mathrm{Aa}$ & $1,82 \mathrm{Aa}$ & $1,72 \mathrm{a}$ \\
\hline 250 & $1,93 \mathrm{Ba}$ & $2,23 \mathrm{Aa}$ & $2,08 \mathrm{a}$ & $1,53 \mathrm{Ba}$ & $1,87 \mathrm{Aa}$ & $1,70 \mathrm{a}$ \\
\hline 500 & $1,93 \mathrm{Aa}$ & $2,02 \mathrm{Aa}$ & $1,98 \mathrm{a}$ & $1,49 \mathrm{Aa}$ & $1,63 \mathrm{Aa}$ & $1,56 \mathrm{a}$ \\
\hline Média & $1,96 \mathrm{~B}$ & $2,14 \mathrm{~A}$ & & $1,54 \mathrm{~B}$ & $1,77 \mathrm{~A}$ & \\
\hline $\mathrm{CV}(\%)$ & \multicolumn{2}{|c|}{7,0} & \multicolumn{4}{|c|}{8,9} \\
\hline
\end{tabular}

${ }^{(1)}$ Médias seguidas por letras iguais, maiúsculas na linha e minúsculas na coluna, não diferem entre si pelo teste de Tukey, a 5\% de probabilidade. ${ }^{(2)}$ Adubação mineral de 0,250 e $500 \mathrm{~kg} \mathrm{ha}^{-1}$ da fórmula 4-14-8.

\section{Conclusões}

1. A adubação orgânica, ao longo dos anos, promove incremento do carbono orgânico total, da estabilidade dos agregados em água, dos teores de $\mathrm{P}$ e de $\mathrm{N}$ nas diferentes classes de agregados, e diminui a relação $\mathrm{C} / \mathrm{N}$ e $\mathrm{C} / \mathrm{P}$.

2. A adubação mineral promove alterações nos teores de P nas diferentes classes de agregados e está relacionada à redução do diâmetro médio ponderado dos agregados, na camada $0-10 \mathrm{~cm}$.

\section{Agradecimentos}

Ao Conselho Nacional de Desenvolvimento Científico e Tecnológico, por bolsa concedida; ao Departamento de Solos da Universidade Federal de Viçosa, pela disponibilização de estrutura física e oportunidade de realização do trabalho.

\section{Referências}

ADESODUN, J.K.; ADEYEMI, E.F.; OYEGOKE, C.O. Distribution of nutrient elements within water-stable aggregates of two tropical agroecological soils under different land uses. Soil and Tillage Research, v.92, p.190-197, 2007.

ASSIS, R.L.; BAHIA, V.G. Práticas mecânicas e culturais de recuperação de características físicas dos solos degradados pelo cultivo. Informe Agropecuário, v.19, p.71-78, 1998.

BASTOS, R.S.; MENDONÇA, E. de S.; ALVAREZ VENEGAS, V.H.; CORREA, M.M. Formação e estabilização de agregados do solo decorrentes da adição de compostos orgânicos com diferentes características hidrofóbicas. Revista Brasileira de Ciência do Solo, v.29, p.11-20, 2005.

BAYER, C.; MIELNICZUK, J. Dinâmica e função da matéria orgânica. In: SANTOS, G. de A.; CAMARGO, F.A. de O. (Ed). Fundamentos da matéria orgânica do solo: ecossistemas tropicais e subtropicais. Porto Alegre: Genesis, 1999. p.9-26.

BREMNER, J.M.; MULVANEY, C.S. Nitrogen total. In: PAGE, A.L. (Ed.). Methods of soil analysis: chemical and microbiological properties. Madison: ASA, 1982. p.595-624.

CARPENEDO, V.; MIELNICZUK, J. Estado de agregação e qualidade de agregados de Latossolos Roxos, submetidos a diferentes sistemas de manejo. Revista Brasileira de Ciência do Solo, v.14, p.99-105, 1990.

DOUGLAS, J.T.; GOSS, M.J. Stability and organic matter content of surface soil aggregates under different methods of cultivation and in grassland. Soil and Tillage Research, v.2, p.155-175, 1982.

EMBRAPA. Manual de métodos de análise de solo. Rio de Janeiro: Embrapa-CNPS, 1997. 212p. (Embrapa-CNPS. Documentos, 1).

FERREIRA, F.P.;AZEVEDO,A.C.de; DALMOLIN, R.S.D.; GIRELLI, D. Carbono orgânico, óxidos de ferro e distribuição de agregados em dois solos derivados de basalto no Rio Grande do Sul-Brasil. Ciência Rural, v.37, p.381-388, 2007.

GALVÃO, J.C.C. Características físicas e químicas do solo e produção de milho exclusivo e consorciado com feijão em função de adubações orgânica e mineral contínuas. 1995. 194p. Tese (Doutorado) - Universidade Federal de Viçosa, Viçosa.

HAYNES, R.J.; SWIFT, R.S. Stability of soil aggregates in relation to organic constituents and soil water content. European Journal of Soil Science, v.41, p.73-83, 1990.

KEMPER, W.D.; CHEPIL, W.S. Size distribution of aggregates. In: BLACK, C.A. (Ed.). Methods of soil analysis. Madison: ASA, 1965. p.499-510. 
LEITE, L.F.C.; MENDONÇA, E.S.; MACHADO, P.L.O.A.; MATOS, E.S. Total $\mathrm{C}$ and $\mathrm{N}$ storage and organic $\mathrm{C}$ pools of a Red-Yellow Podzolic under conventional and no tillage at the Atlantic Forest Zone, Southeastern Brazil. Australian Journal of Soil Research, v.41, p.717-730, 2003.

MATOS, E. da S.; MENDONÇA, E. de S.; VILLANI, E.M. de A.; LEITE, L.F.C.; GALVÃO, J.C.C. Formas de fósforo no solo em sistemas de milho exclusivo e consorciado com feijão sob adubação orgânica e mineral. Revista Brasileira de Ciência do Solo, v.30, p.625-632, 2006.

MURPHY J.; RILEY, J.P. A modified single solution method for the determination of phosphate in natural waters. Analytica Chimica Acta, v.27, p.31-36, 1962.

NEVES, C.S.V.J.; FELlER, C.; KOUAKOUA, E. Efeito do manejo do solo e da matéria orgânica solúvel em água quente na estabilidade de agregados de um Latossolo argiloso. Ciência Rural, v.36, p.1410-1415, 2006.

PALMEIRA, P.R.T.; PAULETTO, E.A.; TEIXEIRA, C.F.A.; GOMES, A.S.; SILVA, J.B. Agregação de um Planossolo submetido a diferentes sistemas de cultivo. Revista Brasileira de Ciência do Solo, v.23, p.189-195, 1999.

PASSOS, R.R.; RUIZ, H.A.; CANTARUTTI, R.B.; MENDONÇA, E. de S. Carbono orgânico e nitrogênio em agregados de um Latossolo Vermelho distrófico sob duas coberturas vegetais. Revista Brasileira de Ciência do Solo, v.31, p.1109-1118, 2007.
PERFECT, E.; KAY, B.D.; VAN LOON, W.K.P.; SHEARD, R.W.; POJASOK, T. Factors influencing soil structural stability within a growing season. Soil Science American Journal, v.54, p.173-179, 1990.

PERIN, A.; GUERRA, J.G.M.; TEIXEIRA, M.G.; PEREIRA, M.G.; FONTANA, A. Efeito da cobertura viva com leguminosas herbáceas perenes na agregação de um Argissolo. Revista Brasileira de Ciência do Solo, v.26, p.713-720, 2002.

SIX, J.; PAUSTIAN, K.; ELLIOTT, E.T.; COMBRINK, C. Soil structure and organic matter: I. Distribution of aggregate-size classes and aggregateassociated carbon. Soil Science Society of American Journal, v.64, p.681$689,2000$.

STEVENSON, F.J. Humus chemistry: genesis, composition, reactions. 2.ed. New York: John Wiley, 1994. 496p.

TISDALL, J.M.; OADES, J.M. Organic matter and water-stable aggregates in soils. European Journal of Soil Science, v.33, p.141-163, 1982.

WENDLING, B.; JUCKSCH, I.; MENDONÇA, E. de S.; NEVES, J.C.L. Carbono orgânico e estabilidade de agregados de um Latossolo Vermelho sob diferentes manejos. Pesquisa Agropecuária Brasileira, v.40, p.487-494, 2005.

YEOMANS, J.C.; BREMNER, J.M. A rapid and precise method for routine determination of organic carbon in soil. Communications in Soil Science and Plant Analysis, v.19, p.1467-1476, 1988.

ZHANG, T.Q.; MACKENZIE, A.F. Changes of soil phosphorous fractions under long-term corn monoculture. Soil Science Society of America Journal, v.61, p.485-493, 1997.

Recebido em 23 de abril de 2008 e aprovado em 12 de agosto de 2008 